\title{
Microstructural Interactions During Stress Ageing a 7475 Aerospace Alloy
}

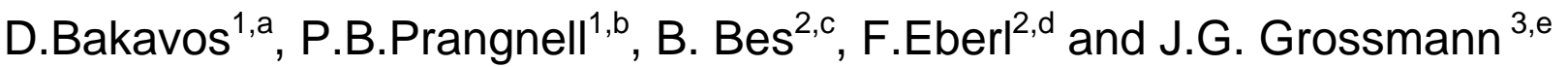 \\ ${ }^{1}$ Manchester Materials Science Centre, UMIST, Grosvenor St. Manchester, M1 7HS, UK. \\ ${ }^{2}$ Alcan Centre de Recherches de Voreppe, 725 rue Aristide Bergès, BP27, 38341 Voreppe Cedex, \\ France. \\ ${ }^{3}$ CCLRC Daresbury Laboratory, Warrington, WA4 4AD, U.K.
}

ad.bakavos@postgrad.manchester.ac.uk, bphilip.prangnell@manchester.ac.uk, cbernard.bes@alcan.com, ${ }^{d}$ Frank.eberl@alcan.com, ej.g.grossmann@dl.ac.uk

Keywords: 7475, Al-Zn-Mg-Cu, Creep, Ageforming, Constant Stress Ageing, Small Angle X-ray Scattering, TEM, GP-I, GP-II Zones, $\eta^{\prime}$, $\eta$, Alignment, Preferential Precipitate Orientation.

\begin{abstract}
In industrial process, like creep-ageforming, materials are aged under load. To investigate the influence of an applied stress on the ageing behaviour of $\mathrm{Cu}$ bearing Al-7xxx series alloys, a 7475 alloy was aged under a constant tensile stress and analysed by means of Small Angle X-ray Scattering (SAXS) and TEM. Mechanical testing was also employed, to determine if there was any effect on the materials strength. The results show that during the early stages of ageing significant interactions takes place, which preferentially aligns one type of GP zone, as well as affecting their size and volume fraction. During the second stage ageing treatment, the applied stress was observed to cause more rapid over-ageing, by promoting the formation of the $\eta$ phase.
\end{abstract}

\section{Introduction}

In certain alloy systems, such as the $2 \mathrm{xxx}$ series with high $\mathrm{Cu}: \mathrm{Mg}$ ratios, interactions caused by the application of a stress during ageing [1-3] have the potential to alter an alloy's microstructure and mechanical properties [5]. Considerable research has been carried out on the effects of an applied stress during ageing in this system (e.g. [1-3]). This work shows that above a critical threshold stress the nucleation of thin plate shaped phases (GP zones, $\theta^{\prime \prime}, \theta^{\prime}$ and $\Omega$ ), with a large plate normalmatrix misfit, are strongly biased onto specific habit plane variants preferentially aligned with the applied stress field. The applied stress can also result in changes in precipitate size and morphology [1-4]. In contrast, hardly any work has been published that has investigated similar phenomena in $7 \mathrm{xxx}$ series alloys. Of particular relevance is the use if these alloys for aerospace applications where they are creep-ageformed, which requires subjecting a material to bending stresses that are subsequently partially relaxed during the application of an artificial ageing treatment. Preliminary studies, carried out by a qualitative TEM investigations of a 7475 alloy in a T73 temper, indicated little obvious effect of stress ageing in this alloy system, with no preferential alignment of the precipitates being observed [3-4]. However, no research was undertaken to clarify if interactions with an applied stress field occurred during earlier stages of aging, such as when the GP zones develop, or if there are subtle effects not immediately apparent by visual examination of TEM micrographs. The focus of the work reported here was, therefore, to investigate in more depth if any type of stess-interaction could be detected in the same 7475 alloy at any stage in a typical T73 ageing treatment.

\section{Experimental Procedure}

The 7475 alloy investigated was supplied as a $60 \mathrm{~mm}$ thick plate in a W351 condition, after solution treatment and stretching by $2 \%$. The material had a grain size of $\sim 170 \mu \mathrm{m}$. Tensile specimens, with a gauge diameter of $8 \mathrm{~mm}$ and length of $55 \mathrm{~mm}$, were machined from the $1 / 4$ and $3 / 4$ thickness positions parallel to the rolling direction. These samples were subjected to a series of constant stress 
ageing treatments at the highest stress level possible without the material failing, of $245 \mathrm{MPa}$, by stopping the test and air cooling after increasing times through a T7351 temper, two-stage, ageing treatment, of $4 \mathrm{hrs}$ at $108{ }^{\circ} \mathrm{C}$, followed by $24 \mathrm{hrs}$ at $160{ }^{\circ} \mathrm{C}$. Stress-free control samples were aged identically by positioning them at the same height in the same furnace. A Philips CM200 TEM was used to characterise the precipitate structures in the aged specimens. The loading direction for the stress-aged samples was marked on the specimen foils before polishing. The precipitates present in the 7475 alloys were further characterised by Small Angle X-ray Scattering (SAXS) experiments at the CCLRC Daresbury Laboratory, UK, (beam line 2.1). After instrument and background corrections the SAXS data was analysed, following the method described in [6], to obtain the Guinier radius $\left(\mathrm{R}_{\mathrm{g}}\right)$, by using an iterative procedure involving fitting the slope of integrated intensity vs scattering vector (I vs $\mathrm{q}^{2}$ ) Kratky plot in the region of the $0.8<\mathrm{qRg}<1.8$. By assuming random alignment of the precipitates, this was converted into a 'mean sphere radius' using the formula; $R_{o}=\sqrt{5 / 3} R_{g}[7]$.

\section{Results}

Stress Aged in final T7351 Temper. After ageing to the full T73 temper, the 7475 alloy contained some $\eta$ in a matrix of a fine distribution of $\eta^{\prime}$ (Fig. 1). The $\eta^{\prime}$ phase develops from GPZs that survive reversion during the first stage of ageing and $\eta$ nucleates at dislocations and dispersoids during the second stage [8-9]. From comparison of the TEM images for the conventionally stressfree (Fig 1(a)) and constant stress-aged samples (Fig. 1(b)), there is no indication of one precipitate variant dominating in any particular direction. In Fig. 1 the intensities of the reflections from the $\eta^{\prime}$ and $\eta$ plate variants (shown in magnified sections of $\langle 111\rangle_{\mathrm{Al}}$ SAD patterns) are symmetrically distributed. In addition to the TEM observations, SAXS patterns from an area detector are shown in Fig. 2(a) and (c) with the average radius $\left(\mathrm{R}_{0}\right.$, determined from each integrated $15^{\circ}$ sector, plotted as a function of angle (Fig.2(b)). Both sets of data have isotropic intensity distributions. Taken together, these results show conclusively that there is no preferred alignment, by preferential habit plane variant selection, of the $\eta^{\prime}$ and $\eta$ phases in the final temper condition, unlike the behaviour seen in Al-Cu-Mg alloys for the $\theta^{\prime}$ and $\Omega$ phases [1-3].

Although there was no preferential alignment of the precipitates at the end of stress-ageing to the T73 temper, from careful comparison of TEM images and diffraction data (Fig. 1(a) and (b)) it was found that the microstructure in the stress-aged alloy was slightly coarser and more dominated by the $\eta$ phase, with a lower density of retained $\eta^{\prime}$. These qualitative TEM observations were confirmed by the SAXS results shown in Fig. 2(b) where, while the sector integrated radii are isotropic, the two sets of results indicate different average particle sizes of $R_{0}=62 \AA$ and $R_{0}=68 \AA$ $( \pm 1 \AA)$ for the stress-free and stress-aged samples, respectively. This suggests that the application of a constant stress during ageing accelerates the precipitation kinetics and results in a coarser more over-aged microstructure, compared to that seen in a conventionally aged alloy.

These microstructural observations have been further confirmed by measuring the yield stress anisotropy from tensile samples machined at different angles to the rolling direction/stress axis after ageing a plate under a constant stress of $210 \mathrm{MPa}$. In Fig. 3 both samples have similar levels of anisotropy, due to texture effects, but there is a systematic small loss in yield strength for the stressaged sample relative to the stress-free aged control $(\sim 6 \%)$. To understand how these differences evolve with time, full ageing curves are shown in Fig. 4, for the stress aged (245MPa) and stressfree samples. These results show small differences between the stress-aged and stress-free curves occur not only in the final temper condition and these effects will be discussed in more detail below.

Influence of an Applied Stress on Under-Ageing. Comparison of the age-hardening curves for the stress-free and stress-aged samples show that slight differences emerge at two stages in the aging responses (Fig. 4). In the as-received naturally aged condition both samples start with the same hardness, but after heating to the first low temperature $\left(108^{\circ} \mathrm{C}\right)$ ageing stage the curves diverge with increasing time at temperature; the stress-aged sample developing a slightly lower 
hardness than the stress-free control. This difference largely disappears on heating to the second higher temperature stage $\left(160^{\circ} \mathrm{C}\right)$ and then develops again with time, due to the more rapid overaging of the stress aged alloy. SAXS analysis has been performed at several stages in this ageing sequence and the results are presented in Fig. 5.
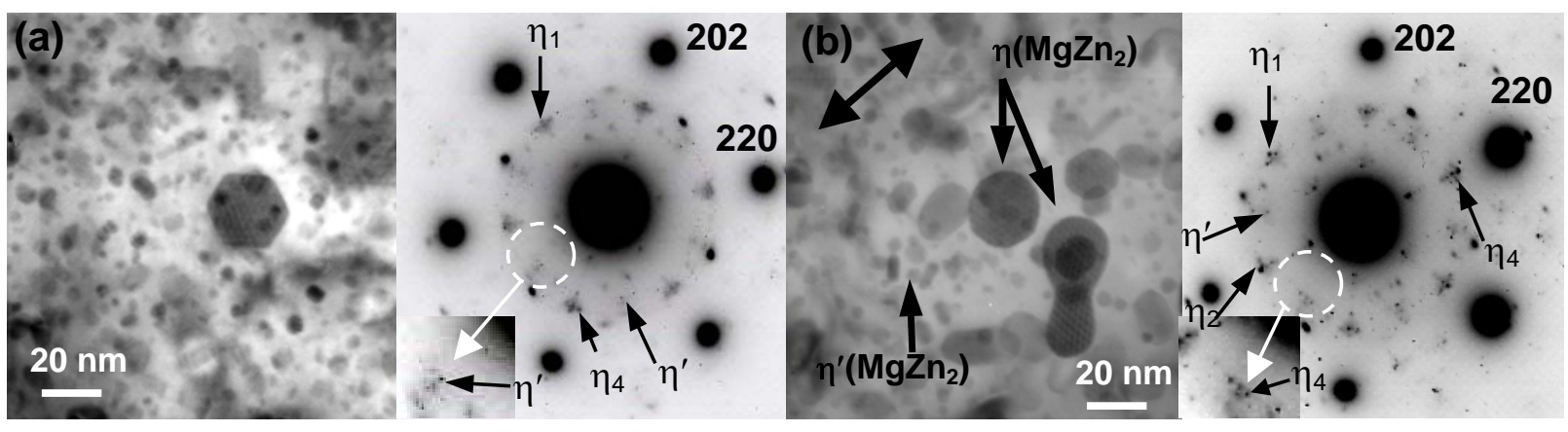

Fig. 1 Bright field (BF) images and $\langle 111\rangle_{\mathrm{Al}}$ selected area diffraction (SAD) patterns from, (a) the conventionally (stress-free) (b) stress-aged (245 MPa) specimens. The double headed arrow in (b) indicates the projected load direction.
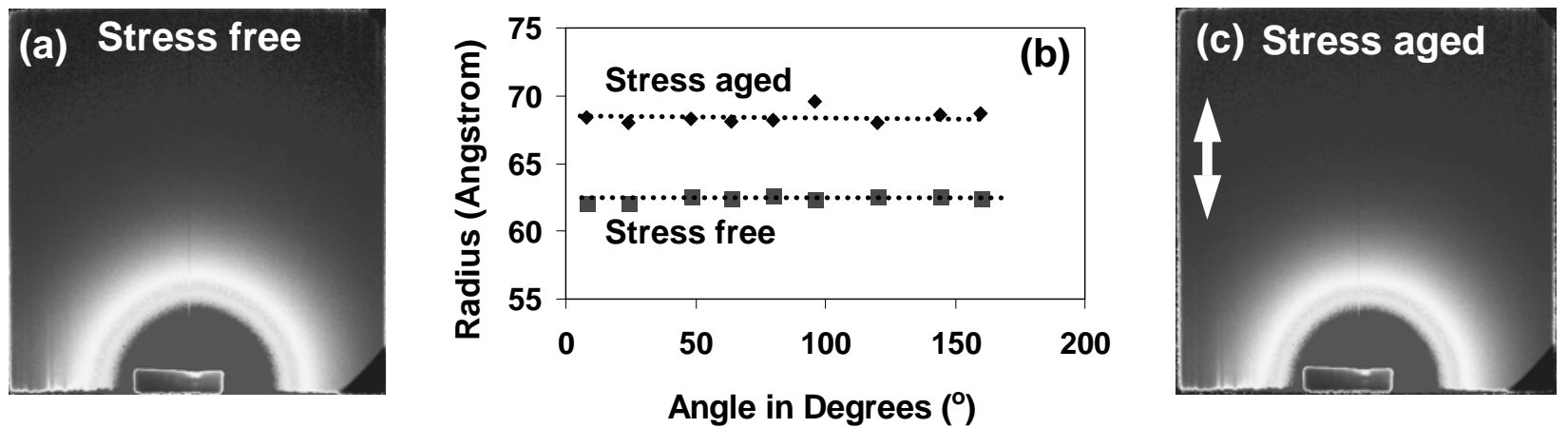

Fig. 2 SAXS patterns from AA7475 aged to the T7351 condition, (a) conventionally and (c) stressaged under a constant $245 \mathrm{MPa}$ tensile stress. (b) shows the sector averaged precipitate radii $\left(\mathrm{R}_{\mathrm{o}}\right.$ for both treatments as a function of angle. The arrow indicates the loading direction $\left(90^{\circ}\right.$ in (b)).

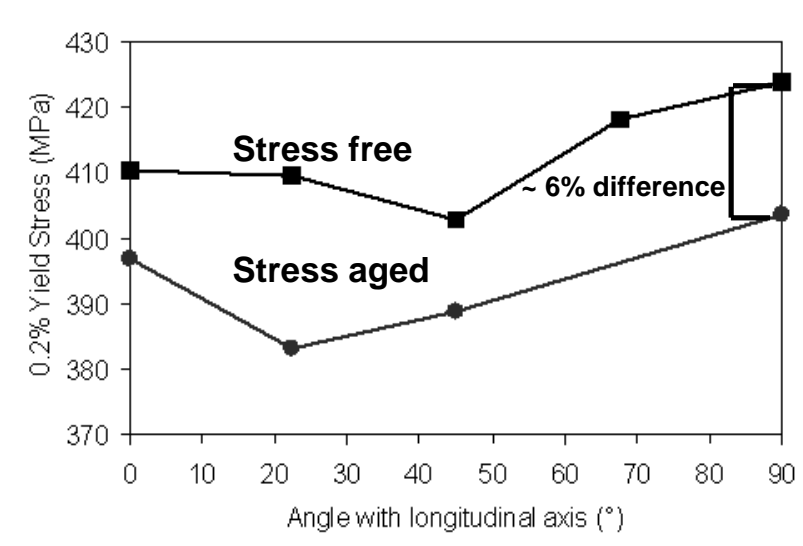

Fig. 3 Yield stress anisotropy measured from tensile samples machined at different angles to the rolling direction/stress axis after ageing a plate under a constant stress of $210 \mathrm{MPa}$, compared to a stress-free control.

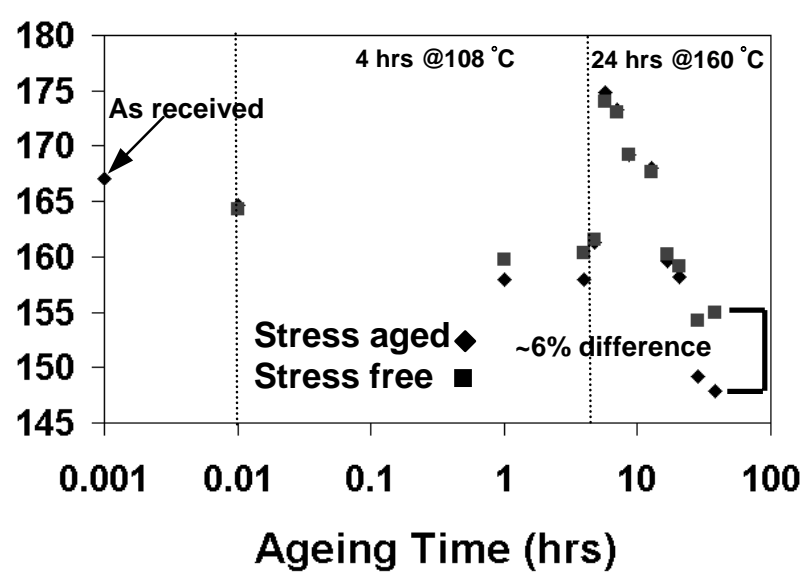

Fig. 4 Age-hardening curves for the two stage temper, comparing ageing under a constant tensile stress of $245 \mathrm{MPa}$ and stress-free ageing. 
(a)
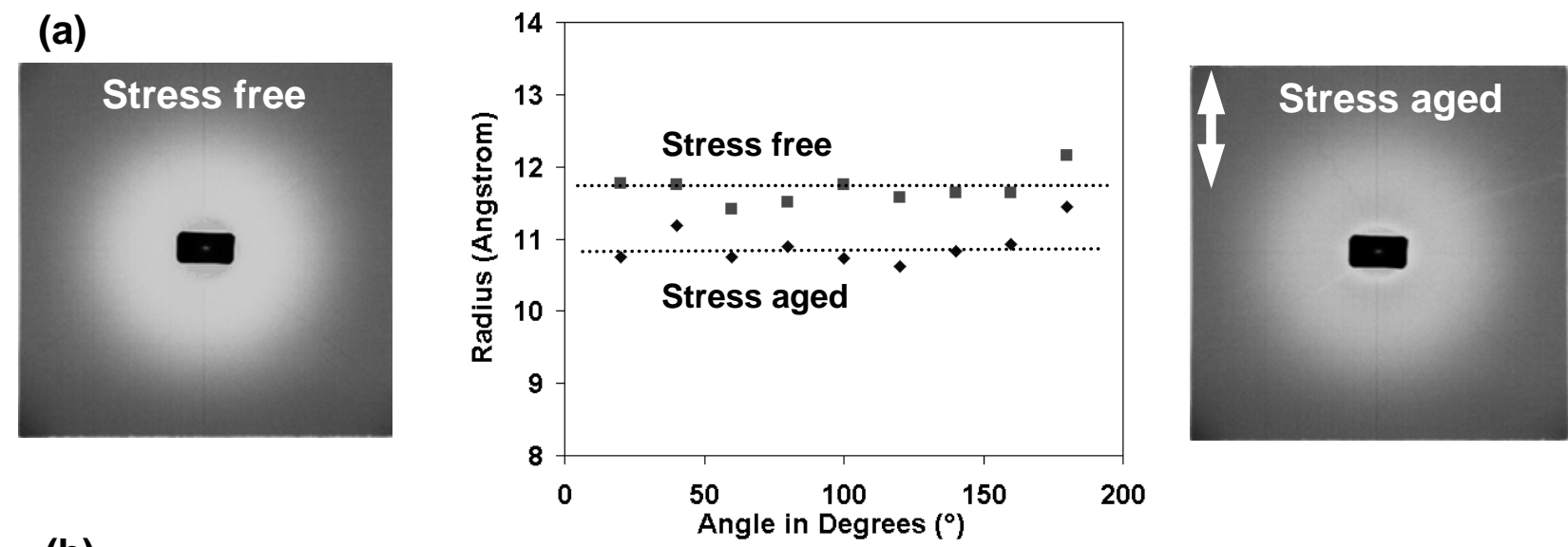

(b)
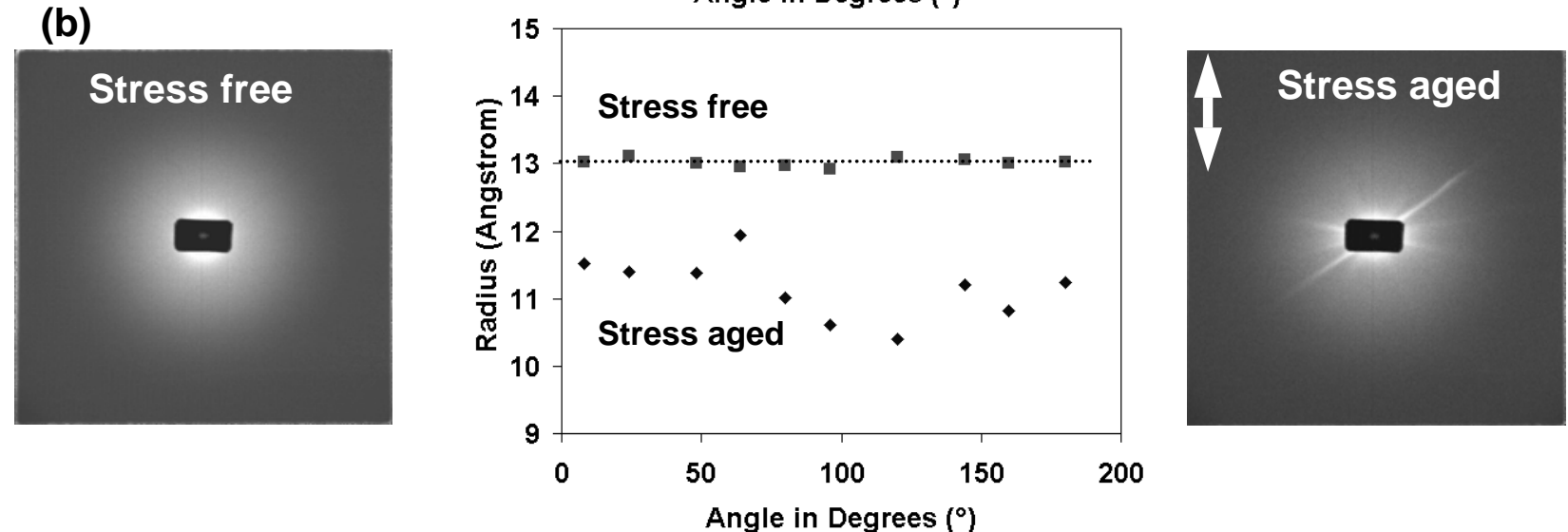

(c)
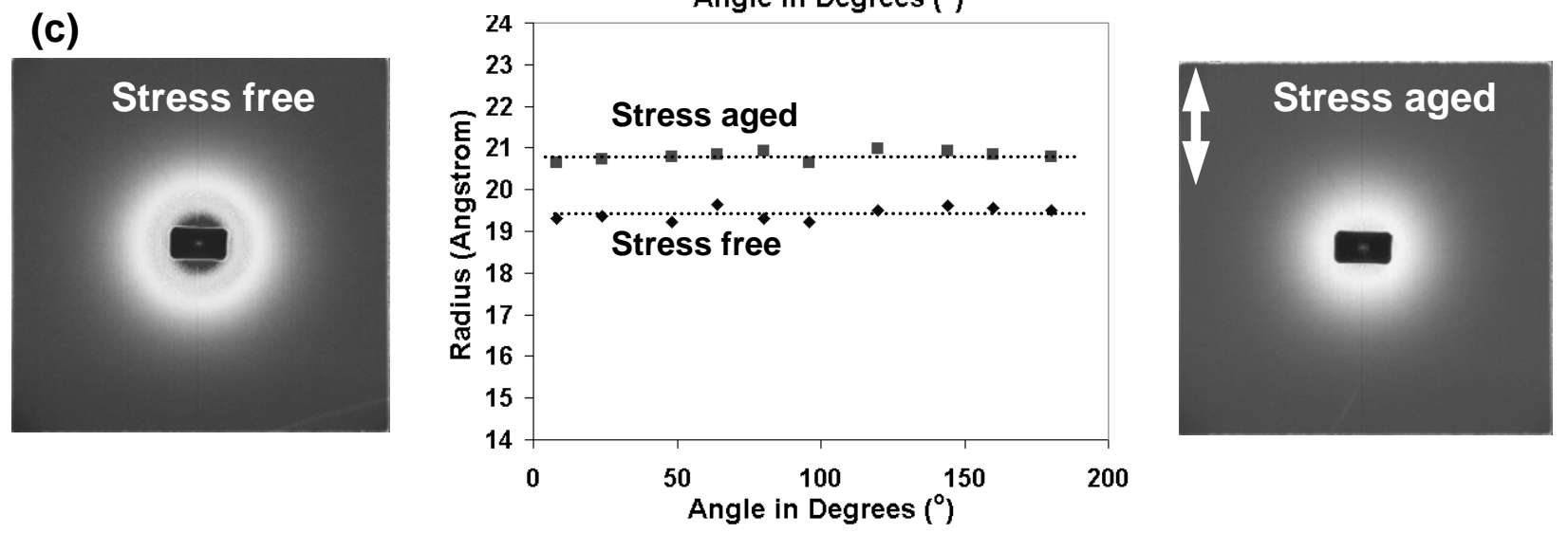

Fig. 5 SAXS results from the conventionally and stress-aged AA7475 alloy (constant $245 \mathrm{MPa}$ tensile stress) for; (a) at the start $\left(0.01 \mathrm{hrs}\right.$ at $\left.108{ }^{\circ} \mathrm{C}\right)$ and (b) the end of the first stage (4hrs @ $108^{\circ} \mathrm{C}$ ) and (c) the top of the second stage ramp $\left(4 \mathrm{hrs} @ 108^{\circ} \mathrm{C}+0.01 \mathrm{hrs}\right.$ at $\left.160{ }^{\circ} \mathrm{C}\right)$. At each stage SAXS patterns are shown along with the average precipitate radii for both treatments as a function of sector angle. The double-headed arrows indicate the loading direction $\left(90^{\circ}\right)$.

End of the first heating ramp $\left(0.01 \mathrm{hrs} @ 108^{\circ} \mathrm{C}\right)$. From the analysis of the SAXS diffraction patterns at the end of the first heating ramp (Fig. 5(a)) it was found that the average scattering intensities were isotropic. This indicates that there is no preferential alignment of the GP zones that would be expected to be present at this stage, and/or that the GPZs are approximately spherical in morphology. This is in agreement with the observations of a several researchers [9-11], that suggest that predominantly low aspect ratio, or spherical, GP-I zones nucleate during natural ageing in Al$\mathrm{Zn}-\mathrm{Mg}-\mathrm{Cu}$ alloys, which revert, or grow, during the first heating ramp [11]. However, a very small difference in the average size of the GPZs was noted for each condition of $R_{0}=14.1 \AA$ and $R_{0}=15.1$ $\AA$ for the stress-free and stress-aged samples, respectively. Furthermore, the scattering intensity was slightly stronger for the stress-free sample, indicating that the GP zone volume fraction was slightly larger compared to the stress-aged specimen. These findings therefore tentatively indicate that there may be a slight microstructural interaction that takes place in the constant stress aged alloy at this 
early stage of ageing, which alters the average size and volume fraction of the GP-zones present, but this effect is too small to significantly alter the hardness values.

End of the first ageing stage (4hrs @108 ${ }^{\circ} \mathrm{C}$ ) After the end of the first ageing stage the SAXS results showed that the difference in the average precipitate size (Fig.5 (b)) became more significant, with a radius $\left(\mathrm{R}_{\mathrm{o}}\right)$ for the conventional and stress-aged sample of $16.8 \AA$ and $14.4 \AA$, respectively. Furthermore, while the intensities in the SAXS diffraction patterns for the stress-free sample were isotropic, the constant stress aged alloy in Fig.5 (b) showed the presence of aligned scattering intensity streaks immerging from a background $\sim$ isotropic intensity distribution. Similar SAXS diffraction intensity patterns with streaks along $\langle 111\rangle_{\mathrm{Al}}$ directions have been observed in other alloy systems, where thin $\{111\}$ habit plane, plate-shaped, precipitates are present in their microstructure [12]. Here, the individual streaks originate from aligned thin domains within single grains from the polycrystalline sample and tended to be grouped at around $50-60^{\circ}$ to the tensile axis. The graph of particle radius $\left(\mathrm{R}_{\mathrm{o}}\right)$ for the stress aged sample in Fig. $5 \mathrm{~b}$, integrated over $15^{\circ}$ sectors and averaged over several SAXS patterns (representing data from $\sim 135$ grains within the material) is also not isotropically distributed and shows a minimum at $90^{\circ}$, which is coincident with the stress axis. At this ageing time the relative integrated intensities for the stress aged sample were found to be significantly lower than for the stress-free sample, with a larger difference than seen at the top of the first heating ramp, suggesting, in line with the hardness measurements, that the volume fraction of the GP-Zones was smaller in the stress-aged alloy.

End of the second heating ramp (4hrs@108 ${ }^{\circ} \mathrm{C}+0.01 \mathrm{hrs} @ 160^{\circ} \mathrm{C}$ ) The SAXS patterns from samples aged to the end of the second heating ramp show that the scattering intensities have changed back to being isotropic (Fig. 5(c)). This result indicates that the preferential alignment of the GP zones observed at the end of the first ageing step diminishes during the second heating ramp, and most of the plate shaped GP-II zones, present at low temperatures in the stress-aged sample, dissolve or transform during heating to the higher ageing temperature $\left(160^{\circ} \mathrm{C}\right)$. The hardness measurements for both stress and stress-free aged samples are also much closer at this stage. The average radius $\left(\mathrm{R}_{\mathrm{o}}\right)$ for the conventional and stress-aged samples were calculated to be $25.1 \AA$ and 26.6 $\AA$, which is also more similar and lies in the size range expected for the $\eta^{\prime}$ precipitate [13].

\section{Discussion}

From the above observations it is concluded that two types of GPZs may be forming in the stress aged condition, spherical GP-I and plate morphology GP-II zones, whereas only GP-I zones are seen in the stress-free aged sample. It has been reported that the spherical zones consist of alternate $\mathrm{Mg}-\mathrm{Zn}$ layers, with a $\{001\}_{\mathrm{Al}}$ habit plane, and the plate shaped zone consists of $\mathrm{Zn}$ and $\mathrm{Cu}$ atoms and has a $\{111\}_{\mathrm{Al}}$ habit plane [9-11]. The plate shaped zone appears to be promoted by the presence of an applied stress that also biases its habit plane variant selection (see Fig.5 (b)). This preferred alignment for the GP-II zone variants appears to be on $\{111\}_{\mathrm{Al}}$, planes tilted at around $\sim 35^{\circ}$ to the tensile axis, which could coincide with $\mathrm{a}<001>_{\mathrm{Al}}$ direction. Why this preferred orientation occurs is not yet fully understood, but implies that the strain energy can be most reduced by relaxing the elastically softer $<001>$ directions, two of which are approximately normal to, and one parallel to, the tensile axis. It is now well established that in $2 \mathrm{xxx}$ alloys the $\theta^{\prime}$ and $\Omega\left(\mathrm{Al}_{2} \mathrm{Cu}\right)$ phases, which have a negative misfit, "prefer" to nucleate on habit planes experiencing a compressive strain as this reduces the strain energy contribution to the energy barrier for nucleation, [1-3]. Evidence from the literature suggests that the plate shaped GP-II zones contain mainly $\mathrm{Zn}$ and $\mathrm{Cu}$ [9-11] and therefore have a higher negative misfit with the matrix than GP-I zones, which is reflected in the different GPZ morphologies. Further work is also required to fully understand why when stress-aged the alloy softens more than the control samples, relative to the naturally aged condition, during this first stage of the ageing treatment, when the aligned GP-II zones develop. Evidence of second phase alignment disappears on ramping to the higher temperature second stage, which coincides with the appearance of $\eta^{\prime}$ and the reversion of the remaining GPZs, and this phase is clearly randomly 
distributed over the available habit plane variants. During the final stage of ageing and the appearance of $\eta$, which is again randomly distributed, the stress aged material overages more rapidly than the control. With a directionally applied stress, a reduction in the energy barrier for nucleation due to a lower net strain energy would naturally result in preferred alignment on habit plane variants orientated so that the nucleus misfit can take maximum advantage of the superimposed stress state. The main effect of the stress cannot, therefore, be directly on the nucleus misfit for the $\eta$ and $\eta^{\prime}$ phases, but must be as a result of dislocation glide and climb activity due to creep under the applied stress. Stretching prior to aging is known to accelerate over-ageing in $7 \mathrm{xxx}$ alloys, by providing dislocation nucleation sites for the equilibrium $\eta$ phase [8]. However, the plastic creep strain is relatively small and therefore the dynamic nature of the precipitation and simultaneous creep processes must have a disproportionate effect on the ageing kinetics.

\section{Summary}

The application of a constant applied stress during artificial ageing a 7475 alloy to a T73 temper has been found to result in subtle, but significant, changes in behaviour. During the early stages of ageing the application of a far-field tensile stress promotes plate shaped GP-II zones. These GP Zones are preferably oriented on $\{111\}$ planes lying $\sim 35^{\circ}$ to the tensile stress axis. However, this effect rapidly disappears during the second ageing stage when the GPZs transform to $\eta^{\prime}$. The $\eta^{\prime}$ and $\eta$ phases that subsequently develop are isotropically distributed, but there is a tendency for more rapid over-ageing during the second stage treatment in agreement with result showed in [4]. This precipitate coarsening can result in a modest loss of strength compared to conventional ageing and occurs as a result of the increased dislocation activity during creep.

\section{Acknowledgements}

This research was carried out under project reference G5RD-CT-2002-00743 "Ageformable Panels for Commercial Aircraft" in the European Commission's fifth framework programme. The authors are also grateful to Dr. K.Geraki (CCLRC Daresbury) for assistance with the SAXS experiments.

\section{References}

[1] B.Skrotzki, G.J.Shiflet, E.A.Starke Jr: Met and Mat. Tra. A, Vol. 27A (1996), p. 3431.

[2] A.W.Zhu, E.A Starke Jr: Act. Mat., Vol. 49 (2001), p. 2285.

[3] D.Bakavos, P.B.Prangnell, R.Dif: Mat. Sci. Forum, Vol . 28 (2004), p. 124.

[4] D.Bakavos, P.B.Prangnell, B.Bes, F. Eberl, S. Gardiner; in the present proceedings.

[5] H.Hargarter, M.T.Lyttle, E.A. Starke: Mat. Sci. and Eng., Vol. A257 (1998), p. 87.

[6] A. Deschamps: Analytical Techniques for aluminium alloys, Handbook of Aluminium, Vol. 2, (Marcel Dekker, Inc., New York, USA, 2003, ed.G.E. Totten and D.S.MacKenzie), p.155.

[7] M.R.Baig, S.Gupta, S.Messoloras and R.J.Stewart: J.Appl.Cryst. Vol. 24 (1991), p. 349.

[8] A.Deschamps, Y.Brechet: Mat. Sci. and Eng. Vol. A47 (1999), p. 281.

[9] T.Engdhal, V.Hansen, P.J.Warren, K.Stiller: Mat. Sci. and Eng. Vol. A327 (2002), p. 59.

[10] K.Stiller, P.J.Warren,V.Hansen, J.Angnete, J.Gjones: Mat. Sci. and Eng.Vol.270 (1999), p. 55.

[11] N.Qchinh, J.Ledvai, D.H.Ping, K.Hono: J. of Alloys and Compounds. Vol. 378 (2004), p. 52.

[12] M.Kompatscer, M.Schonfeld, B.Heinric, H.Kostorz: J.Appl.Crys. Vol.136 (2000), p. 397.

[13] M.Dumont,W.Lefebvre, B.D.Cottignes, A.Deschamps: Acta Mat. Vol. 53, (2005), p. 2881. 